Research Paper

\title{
Hepatoprotective effects of limb ischemic post-conditioning in hepatic ischemic rat model and liver cancer patients via PI3K/ERK pathways
}

Yanfeng Gao ${ }^{*}$, Shuang Zhou ${ }^{2,3^{*}}$, Fengfei Wang $2,3,4,5^{*}$, Yue Zhou ${ }^{6}$, Sen Sheng7, Dan Qi2,3, Jason H. Huang ${ }^{2,3,4}$, Erxi Wu ${ }^{2,3,4,8,9 凶}$, Yi Lv ${ }^{10 \bowtie}$, Xiongwei Huo ${ }^{11 \bowtie}$

1. Department of Anesthesiology, the First Affiliated Hospital of Xi'an Jiaotong University, Xi'an 710061, China;

2. Department of Neurosurgery, Baylor Scott \& White Health, Temple, Texas 76502, USA;

3. Neuroscience Institute, Baylor Scott \& White Health, Temple, Texas 76502, USA;

4. Department of Surgery, Texas A \& M University Health Science Center, College of Medicine, Texas 76508, USA;

5. Department of Neurology, Baylor Scott \& White Health, Temple, Texas 76502, USA;

6. Department of Statistics, North Dakota State University, Fargo, North Dakota 58105, USA;

7. Department of Neurology, University of Arkansas for Medical Science, Little Rock, Arkansas 72205, USA;

8. Department of Pharmaceutical Sciences, Texas A \& M University Health Science Center, College of Pharmacy, College Station, Texas 77843, USA;

9. LIVESTRONG Cancer Institutes, Dell Medical School, the University of Texas at Austin, Austin, Texas 78712, USA;

10. Department of Hepatobiliary Surgery, the First Affiliated Hospital of Xi'an Jiaotong University, $X_{i}$ an 710061, China;

11. Department of General Surgery, the First Affiliated Hospital of Xi' an Jiaotong University, Xi' an 710061, China.

*These authors contribute equally to this study

$\square$ Corresponding author: Erxi Wu, Department of Neurosurgery, Baylor Scott and White Health, Temple, Texas, 76502, USA. Email: Erxi.wu@bswhealth.org; Yi Lv, Department of Hepatobiliary Surgery, the First Affiliated Hospital of Xi'an Jiaotong University, Xi'an, 710061, China. Email: luyi168@126.com; Xiongwei Huo: Department of General Surgery, the First Affiliated Hospital of Xi'an Jiaotong University, Xi'an, 710061, China. Email: doctorhuoxw@163.com

(1) Ivyspring International Publisher. This is an open access article distributed under the terms of the Creative Commons Attribution (CC BY-NC) license (https://creativecommons.org/licenses/by-nc/4.0/). See http://ivyspring.com/terms for full terms and conditions.

Received: 2018.07.10; Accepted: 2018.10.05; Published: 2018.11.03

\begin{abstract}
The most effective way of treating liver cancer is surgical resection, which usually requires blocking the hepatic portal circulation, and may result in hepatic ischemia-reperfusion injury (HIRI). It is of paramount importance to control HIRI for liver cancer surgical resection. In this study, a $70 \%$ ischemia-reperfusion $(I / R)$ model of rat liver was established, and the protective effect and mechanism of limb ischemic post-conditioning (LIPOC) on HIRI was investigated. We show that LIPOC has a protective effect on hepatic ischemia-reperfusion injury in rats, which reduces the elimination of superoxide dismutase, thereby increasing oxygen free radical scavenging, decreasing lipid peroxidation, inhibiting neutrophil aggregation, as well as reducing TNFa, ILI $\beta$, and other inflammatory cytokines. In addition, LIPOC inhibited the apoptosis of hepatocytes induced by $\mathrm{I} / \mathrm{R}$ injury, and decreased the $\mathrm{Bax} / \mathrm{Bcl}-2$ ratio. Furthermore, LIPOC promoted the phosphorylation of Akt and ERK1/2. The use of PI3K inhibitor LY294002 and ERK1/2 blocker PD98059 inhibited the phosphorylation of Akt and ERK1/2 caused by LIPOC and abolished the injury protection of liver I/R. Moreover, through 16 cases of hepatocellular carcinoma resections, we found that short-term LIPOC treatment significantly suppressed the elevated alanine aminotransferase, aspartic transaminase, and total bilirubin in the early post-operation of liver resection, and reduced reperfusion injury to the ischemic liver. In summary, our study demonstrates that LIPOC could be an effective method for HIRI in the clinical implementation of liver resection and uncovers the potential mechanism of LIPOC in the protective effects of HIRI.
\end{abstract}

Key words: limb ischemic postconditioning (LIPOC), hepatic ischemia-reperfusion injury, liver cancer, hepatectomy, reperfusion injury salvage kinase

\section{Introduction}

Liver cancer is one of the most common and deadly malignancies worldwide. In recent years, incidence increases rapidly by about $3 \%$ per year in women and $4 \%$ per year in men [1]. Currently, the 
most effective approach to treat patients with liver cancer is surgical resection. Liver surgery requires blocking the hilus hepatis, for which a Pringle method [2] is often used. However, this method could result in many surgical complications and side effects such as liver ischemia, intestinal bleeding and reperfusion, and other pathophysiological processes, which subsequently leads to hepatic ischemiareperfusion injury (HIRI) and limits the clinical practice of liver resection as well as other related surgeries. Therefore, it is necessary to understand the underlying mechanism of HIRI, find a suitable preventive strategy to minimize HIRI during hepatectomy, extend the tolerance time of liver heat, reduce liver damage, protect liver function, and prevent liver failure.

Recently, more and more studies have confirmed that ischemic preconditioning (IPC) of the organ is an effective HIRI protective approach [3-6]. However, its necessity for being carried out before the organ ischemia makes it difficult to implement in clinical practice. Oxman et al. discovered a method by IPC in the lower limbs namely limb ischemic pre-conditioning (LIPC) that has a protective effect for myocardium in the ischemia/reperfusion (I/R) process [7]. Compared with the classic IPC, LIPC is a simple and non-invasive method with little impact to surgical operation and without extending the operation time, though must be carried out before organ ischemia [8-10]. In comparison, ischemic post-conditioning (IPO) was found to be an important protective mechanism for ischemia, and Zhao et al. demonstrated that IPO has myocardial protective effects using a canine myocardial infarction model [11], though still requires invasive operation. In 2005, Kerendi et al. first reported that a method of remote ischemic post-conditioning (RIPOC) performs 5-min ischemia/ 1-min reperfusion on kidney before myocardial reperfusion to reduce the infarct size of myocardial ischemia-reperfusion injury (IRI) [12]. However, this treatment results in renal ischemia and damages renal function; thus, it is limited in clinical application [12]. Recently, researchers discovered an improved RIPOC method based on the principles of LIPC and RIPOC. By using this post-conditioning limb ischemia, the myocardial and cerebral protective effects have been improved [13-16]. This improved RIPOC is also known as limb ischemic post-conditioning (LIPOC) which is a simple and non-invasive method without influencing operation procedures or extending the operation time. More importantly, it can be carried out after organ ischemia, and therefore, provides the technical and rational basis for clinical application and has significant clinical value. The cardioprotective effect of LIPOC has been demonstrated in animal models and human trials [13, 16]; however, whether LIPOC with protective effect on the liver has not yet been clarified.

When I/R happens, several kinases are activated to inhibit cell apoptosis, which are known as reperfusion injury salvage kinases (RISKs) [17, 18]. This includes phosphatidylinositol-3-kinase/protein kinase B (PI3K/Akt) pathway and extracellular signal-regulated kinase1/2 (ERK1/2) pathway. Studies have shown that overexpression of these kinases has a protective effect on reperfusion myocardium for both ischemic preconditioning and post-conditioning [18-21]. Also, the RISKs are recruited to function in the protection of heart, brain, and liver by IPO, LIPC and RIPOC [22-27]. Pharmacological post-conditioning was shown to protect liver against HIRI via the activation of RISK pathways [28]. However, whether the RISK pathways are involved in the protective effect of LIPOC on HIRI has not been cleared yet.

To address these issues, we established a $70 \%$ liver I/ $\mathrm{R}$ model in rats to explore the protective effects of LIPOC on HIRI and the underlying mechanism. Moreover, the protective effects of LIPOC on liver were also investigated in 16 primary liver cancer patients with hepatectomy. Therefore, this study elucidates the potential mechanism of LIPOC in the protective effects of HIRI and provides evidence showing that LIPOC can be a valuable effective method for HIRI in the clinical implementation of liver resection.

\section{Materials and Methods}

\section{Animal Model}

Male Sprague-Dawley (SD) rats were obtained from the Animal Center of School of Medicine, Xi'an Jiaotong University. Animal experiments were performed according to the National Institute of Health Guide for the Care and Use of Laboratory Animals, and the animal operation protocol was approved by the Animal Ethics Committee of School of Medicine, Xi' an Jiaotong University. Animals were fasted for $12 \mathrm{~h}$ before surgery. Sodium pentobarbital $(3 \%)$ was injected $(40 \mathrm{mg} / \mathrm{kg})$ intraperitoneally for anesthesia. The model of $70 \%$ hepatic ischemia was then established based on previously published methods $[29,30]$. The median incision was made in the upper abdomen. The middle lobe and the right lobe of the liver were separated, followed by the separation of the left lobe and the caudate lobe. We could then observe the hepatic left artery, portal vein, and bile duct running together for about $0.5 \mathrm{~cm}$, and then splitting into their respective lobe. By slightly separating the posterior hepatic vein from anterior 
wall, we then clamp or loose hepatic artery, portal vein, and bile duct using non-invasive small blood vessel clamp in the common running section without separating them individually. After blocking the running for $60 \mathrm{~min}$, the establishment of nearly $70 \%$ of the liver with ischemia was achieved. Evidence of ischemia was based on visualizing the pale blanching of the ischemic lobes. The right lobe and caudate lobe of liver blood flow were not blocked to avoid gastrointestinal congestion. For PI3K inhibitor and ERK1/2 blocker administration groups, $0.3 \mathrm{mg} / \mathrm{kg}$ LY294002 (Sigma) or 5.0 mg/kg PD98059 (Sigma) was intravenous administrated before abdominal closure, respectively.

\section{IPO and LIPOC Treatment}

For IPO groups, after the $70 \%$ hepatic ischemic model was established, IPO was achieved by 10 -sec reperfusion followed by 10 -sec re-occlusion for six times at the onset of reperfusion after warm ischemia as previously described [11]. LIPOC treatment was established as previously described [31]. Briefly, after the $70 \%$ hepatic ischemic model was established, 20 $\mathrm{cm}$ long elastic tourniquet (2 $\mathrm{mm}$ in diameter) was used to bundle the lower limbs at the upper $1 / 3$ parts. The distal surface skin temperature decreased and the color became purple, which were used as signs of successful ischemia in the lower extremities. When loosening the tourniquet to restore lower limb blood flow after reperfusion, the color of the bundled distal limb area restored rosy.

\section{Serum Alanine Aminotransferase (ALT) and Aspartic Transaminase (AST) Measurement}

The rats were sacrificed at $6 \mathrm{~h}$ after long-time reperfusion. Blood samples were collected from sub-hepatic vena cava. The serum levels of ALT and AST were measured by clinical automated chemistry analyzer (Hitachi 7170A). An increase in pyruvate concentration corresponds with the levels of AST and ALT activities in the presence of their substrates L-aspartate or L-alanine, respectively. The pyruvate concentration was determined spectrophotometrically in the form of hydrazine for absorbance at $510 \mathrm{~nm}$.

\section{Malondialdehyde (MDA) Measurement}

In the sacrificed animals, liver tissue samples were harvested and homogenized at $4^{\circ} \mathrm{C}$ into $10 \%$ homogenization, which was used for the measurement of MDA levels. The content of MDA was assessed using thiobarbituric acid (TBA) assay kit (Nanjing Jiancheng Research Institute) according to manufacturer's instruction. TBA can react with MDA that is produced during the degradation of lipid peroxides, which generates a substrate with a strong absorption peak at $532 \mathrm{~nm}$. The levels of MDA in the tissues (nmol/mg) was calculated as follows:

MDA in tissue $(\mathrm{nmol} / \mathrm{mg})=\left[\left(\right.\right.$ Absorbance $_{\text {sample tube }}-$ Absorbance $\left._{\text {blank tube }}\right) /\left(\right.$ Absorbance standard tube - $_{\text {- }}$ Absorbance blank tube) $)$ x $10 \mathrm{nmol} / \mathrm{ml} /$ protein concentration $(\mathrm{mg} / \mathrm{mL})$.

\section{Superoxide Dismutase (SOD) Activity Analysis}

The SOD activity was measured using xanthine oxidase assay kit (Nanjing Jiancheng Research Institute) according to manufacturer's instruction. Xanthine interacts with xanthine oxidase to produce oxygen free radicals, which oxidizes hydroxylamine to form nitrite. Nitrite then reacts with a chromogenic agent to generate purple color that can be measured by visible light spectrophotometer. SOD inhibits oxygen free radicals and reduces the absorbance. Liver tissue samples harvested from sacrificed animals were homogenized and used for the measurement of SOD activities. The amount of SOD for $50 \%$ inhibition was defined as one SOD unit (U), and SOD enzyme activity was expressed as $\mathrm{U} / \mathrm{mg}$ tissue protein.

\section{Myeloperoxidase (MPO) Activity Analysis}

MPO exists in polymorphonuclear leukocytes (PMN). The MPO activity was measured using hydrogen peroxide $\left(\mathrm{H}_{2} \mathrm{O}_{2}\right)$ assay kit (Nanjing Jiancheng Research Institute) according to manufacturer's instruction. $\mathrm{MPO}$ can reduce $\mathrm{H}_{2} \mathrm{O}_{2}$ during reaction, and its enzyme activity can be quantitatively determined by evaluating the amount of PMN. By reacting with the hydrogen donor o-anisidine, it generates yellow product. By colorimetric determination of the product formation at $460 \mathrm{~nm}$, the activity of $\mathrm{MPO}, \mathrm{H}_{2} \mathrm{O}_{2}$ reduction, and the amount of white blood cells can be assessed. The amount of MPO to decompose $1 \mu \mathrm{mol}_{2} \mathrm{O}_{2}$ per gram wet tissue sheet at $37^{\circ} \mathrm{C}$ was defined as one enzyme activity unit, and expressed as $\mathrm{U} / \mathrm{g}$ tissue.

\section{ELISA Assay}

Blood samples were collected from sub-hepatic vena cava of rats at $6 \mathrm{~h}$ after long-time reperfusion. Rat TNF-alpha Immunoassay and Rat IL-1 $\beta$ Immunoassay (Shanghai Langka Biotechnology) are ELISA assays designed to measure rat TNF-alpha and IL-1 $\beta$ levels in cell culture supernatants, serum, and plasma. The ELISA assays were performed according to manufacturer's instruction.

\section{Immuno-histochemistry Staining}

$2.0 \mathrm{~cm} \times 1.0 \mathrm{~cm} \times 0.3 \mathrm{~cm}$ size tissues in the middle part of the middle hepatic tissue from rats were cut for staining. Immunohistochemistry was 
performed using SP immunohistochemistry kit (Nanjing Jiancheng Research Institute, China) and antibodies against Bcl-2 and Bax (Bioss Antibodies, China) were used according to the manufacturer's instructions. The results were visualized using Olympus VanoxT AH-2 light microscope (Olympus, Japan). Brown-yellow granules in the nuclear membrane and cytoplasm of hepatocytes were considered as positive expression of Bcl-2 and Bax. A computerized image system was used to analyze the images. Under the microscope, five random view fields were selected for each section. The total number of hepatocytes and the number of positive cells in each field were counted. The protein expression index (PEI) in the tissues was calculated by the number of positive cells / total number of cells in visual field and presented as percentage.

\section{H\&E Staining}

The tissues from left part of the rat liver were fixed with $10 \%$ neutral formalin, embedded in paraffin, and stained with hematoxylin-eosin (H\&E). Two micrometer thick sections were mounted on glass slides, dewaxed with xylene and rehydrated in a descending alcohol row. Immersed in Harris hematoxylin dye solution for $8 \mathrm{~min}$ and then rinsed in $1 \%$ HCL / 70\% ethanol solution for 5-10 seconds. After rinsing with $\mathrm{H}_{2} \mathrm{O}$, the tissues were stained with eosin for $3 \mathrm{~min}$. The sections were then dehydrated in an ascending alcohol row and rinsed with xylene followed by neutral resin sealing. The specimens were observed under $\mathrm{CHZO}$ light microscope (Olympus).

\section{Quantification of Apoptotic Cells Using TUNEL Staining}

Terminal deoxynucleotidyltransferase-mediated UTP end labeling (TUNEL) assay was performed on 4 $\mu \mathrm{m}$ paraffin-embedded sections of liver tissues. The sections were deparaffinized in Xylenes (Sigma) $(2 \times 8$ $\mathrm{min}$ ) and rehydrated through a descending alcohol row. The samples were incubated in $3 \% \mathrm{H}_{2} \mathrm{O}_{2}$ at room temperature for $10 \mathrm{~min}$ and washed in $0.01 \mathrm{M}$ TBS $(3 \times$ $3 \mathrm{~min})$. After digestion with fresh made TBS solution with protease $\mathrm{K}$ added by $1: 200$ at $37^{\circ} \mathrm{C}$ for $15 \mathrm{~min}$, the samples were treated with the terminal transferase mix [terminal deoxytransferase enzyme (TdT), TdT buffer, biotin-16-dUTP, and $\mathrm{H}_{2} \mathrm{O}$ ] and incubated at $37^{\circ} \mathrm{C}$ for $2 \mathrm{~h}$ in humid air. The reactions were terminated by adding the TdT reaction termination buffer for $30 \mathrm{~min}$ and washing with TBS $(3 \times 3 \mathrm{~min})$. The nuclei that had incorporated the biotinylated dUTP were detected using DAB kit (Bioss Antibodies, China). The samples were washed in $1 \times$ PBS $(2 \times 3$ min), dehydrated through sequential steps of $70 \%$ and $100 \%$ ethanol (3 min each), dried, and mounted.
Counts of the apoptotic cells were performed using a light microscope at $\times 400$ magnification. Five random view fields were selected for each section. The total numbers of apoptotic hepatocytes in each 100 hepatocytes were counted. The Morphology of apoptotic cells in line with: no inflammatory reaction around the cell, membrane shrinkage, nucleus dense and deeply stained as brown fragments or particles. The staining results were quantified using ImageJ software with the Immunohistochemistry (IHC) Image Analysis Toolbox plugin. The percentage of apoptotic cells (TUNEL staining positive) were expressed as apoptosis index (AI). PEI was calculated by the number of positive stained cells (brown staining) / total number of cells in visual field and presented as percentage. The measurement data were expressed as mean \pm standard deviation.

\section{Electron Microscopy}

For electron microscopy, the tissues from left part of the rat liver were cut into $1 \times 1 \times 1 \mathrm{~mm}^{3}$ cubes, fixed with fresh pre-cooled $2.5 \%$ glutaraldehyde for more than $2 \mathrm{~h}$ at $4^{\circ} \mathrm{C}$, followed by incubation in $0.1 \mathrm{M}$ phosphoric acid buffer for $30 \mathrm{~min}$ and fixed with $1 \%$ osmium acid at $4^{\circ} \mathrm{C}$ for $2 \mathrm{~h}$ in the dark. The tissues were then rinsed with $0.1 \mathrm{M}$ phosphoric acid buffer for $10 \mathrm{~min}$ and dehydrated in an ascending alcohol row, and stained with ethanol acetic acid uranyl oxide block for $2 \mathrm{~h}$. After rinsing in $90 \%$ and $100 \%$ ethanol and propylene oxide, the tissues were immersed and embedded with epoxy resin Epon812. Thin sections $(50-70 \mathrm{~nm})$ were cut and stained with uranyl acetate and lead citrate. The specimens were observed under JEM-100SX transmission electron microscope (JEOL USA, Inc.).

\section{RT-qPCR Analysis}

Total RNA was isolated from liver tissue from rats using TRIzol Reagent (Invitrogen) by following the manufacturer's protocol. The quantity and quality of isolated RNA were determined using NanoDrop 1000 spectrophotometer (Thermo Scientific). One microgram of total RNA was used to prepare cDNA using RevertAidTM First Strand cDNA Synthesis Kit (Fermentas Life Sciences) by following the instructions provided by the manufacturer. Primers used in this study are synthesized by Sangon Biotech. The sequences are as follows: Akt forward: 5'-TCATTGAGCGCACCTTCCAT-3'; Akt reverse: 5'-CTCCTGCCGTTTGAGTCCAT-3'; Erk1 forward: 5'-AACCCAAACAAGCGCATCAC-3'; Erk1 reverse: 5'-AAGGTGAATGGCTCCTCAGC-3'; Erk2 forward: 5'-GGTTGTTCCCAAACGCTGAC-3'; Erk2 reverse: 5'-ATACTGCTCCAGGTACGGGT-3'. The original cDNA reaction mixture was diluted to one-tenth of 
the reaction volume. Two microliter of the diluted cDNA was used as the template in the quantitative PCR reaction. PCR amplification was performed using SYBY Premix Ex TaqTM II (TaKaRa Biotechnology) and CFX96 Touch $^{\text {TM }}$ Real-Time PCR Detection System (Bio-Rad).

\section{Western Blotting}

One gram liver tissues from rats were homogenized using Tris-Triton cell lysis buffer (TTL buffer, 1\% Triton X-100, $50 \mathrm{mM}$ Tris- $\mathrm{HCl} \mathrm{pH}$ 7.4, 5\% glycerol, $100 \mathrm{mM} \mathrm{NaCl}$ ) supplemented with protease and phosphatase inhibitor cocktails (Abcam). After adjusted to the same concentration, samples were boiled in SDS-PAGE sampling buffer, analyzed by $10 \%$ SDS-PAGE, and transferred onto the PVDF membrane. Immunoblots were probed with antibodies against Akt, phosphor-Akt (Ser473), Erk1/2 or phosphor-Erk1/2 (Thr408) (Cell Signaling Technology) and reprobed with anti- $\beta$-actin antibody (Sigma) to serve as a loading control. Signals of targeted proteins were detected by the Immun-Star HRP peroxide Luminol/ Enhancer (BIO-RAD) and recorded on CL-XPosure Film (Thermo Scientific). The gray scale of the band was measured by Quantity One image analysis software (Bio-Rad) and the relative expression of the target protein was calculated with the net index (NI) of the $\beta$-actin. The relative expression of the target protein $=$ target protein gray value / $\beta$-actin gray value. The level of phosphorylation was expressed as a percentage of p-Akt / Akt or p-ERK1/2 / ERK1/2 gray values, respectively.

\section{Human Subjects}

Patients underwent liver cancer resection from June 2013 to December 2013 in First Affiliated Hospital of Xi'an Jiaotong University for randomized controlled prospective clinical studies were selected. The human subject studies were approved by the ethical standards committee of $\mathrm{Xi}^{\prime}$ an Jiaotong University. Written informed consents were obtained from the patients participating in the study. Sixteen patients with primary liver cancer, aged 28 to 65 years, 14 males and 2 females were included. All these patients were ASA I-II grade without other diseases in any important organs and with no ongoing radiotherapy and chemotherapy; all patients had no historic liver surgery and peripheral vascular disease affecting their lower limb function (Supplemental Table 1).

The included patients were randomized into two groups: the control group and the LIPOC group, eight in each group. LIPOC consisted of two 4-min tourniquet binding/4-min reperfusion cycles by automated cuff-inflator placed on the right leg after occlusion of hepatic hilum; while in control group, the right leg blood-flow was not blocked. The patients' venous blood samples were collected for the detection of liver function (including ALT, AST, TBIL, and ALB) before operation, at the end of surgery and at 1 day after operation.

\section{Statistical Analysis}

SPSS 13.0 statistical software was used for all statistical analyses of this study. The measurement data were expressed as mean \pm standard deviation. Comparison between groups was performed using analysis of variance, and two pairs of comparison were using SNK-q test; when the variance was not uniform, comparisons were performed using rank sum test. $P<0.05$ for the difference was considered statistically significant.

\section{Results}

\section{LIPOC ameliorates hepatic ischemia-reperfusion injury}

The rat model of hepatic ischemia-reperfusion was first established (Fig. 1A), and 24 healthy male Wistar rats were randomly divided into 4 groups: sham-operated group $(S)$, ischemia-reperfusion group (I/R), ischemic post-conditioning group (IPO) and limb ischemic post-conditioning group (LIPOC). In $\mathrm{S}$ group, duodenohepatic ligament was dissected only. One hour inflow occlusion was performed in I/R group without additional intervention. IPO was achieved by 10 -second reperfusion followed by 10 -second re-occlusion for six times at the onset of reperfusion after warm ischemia. In LIPOC group, both hind limb roots were occluded for 5 min with a tourniquet and released at $1 \mathrm{~min}$ before liver reperfusion (Fig. 1B). The rats were sacrificed at $6 \mathrm{~h}$ after long-time reperfusion. The pathological changes in liver section were observed under light microscope (Fig. 1C) and electron microscope (Fig. 1D).

As shown by the light microscopy image in Fig. 1C, in the S group, the cytoplasm of hepatocytes and sinusoidal sinusoids were normal, while the structures of hepatic lobule, central vein and portal area were clear with no infiltration of inflammatory cells. In the I/R group, liver cell cord arrangement disorder, hepatocyte and sinusoidal endothelial cell swelling, edema and degeneration, hepatic lobular central vein and liver sinus congestion, and liver sinus stenosis were observed. The surrounding part of the liver cells showed vacuolar change. Liver lobular hepatic necrosis was significant, while PMN aggregation and infiltration were observed in the liver tissue (Fig. 1C). In the IPO group, the hepatic 
congestion was attenuated, the structure of hepatic lobule was normal, and the structure of hepatocellular cords, centrilobular central vein and hepatic sinusoids were clear. The hepatocytes swelling and cell degeneration were not obvious. In comparison, in the LIPOC group, the hepatic injury was significantly lightened than that in the I/R group. The central venous structure of the hepatic portal region was normal and no obvious deformation observed. The structure of the hepatocyte cord was clear. The hepatocytes were swollen, but with no necrosis (Fig. 1C).

Electron microscopy images (Fig. 1D) showed that the structure of liver cells in S group was normal, with the morphology of nuclei was regular, the distribution of chromatin was uniform, and the structure of mitochondria was normal. In the I/R group, the structure of hepatocytes was unclear, the nuclear envelope was thickened. In the cytoplasm, there were many circular inclusion bodies with high electron density containing necrotic mitochondria. The nucleus was round (Fig. 1D). In the IPO group, the clarity of the hepatocyte structure was poor. Some nuclei were round and the nucleolus was clear. The chromatin distribution in the nucleus was uniform (Fig. 1D). In the LIPOC group, the structure of liver cells was poorly defined. The nuclei were rounded, the nucleolus was obvious, the nuclei chromatin was distributed evenly (Fig. 1D). Therefore, the histologic changes of the liver tissues under both light microscope and electron microscope were more obvious in I/ $R$ group than in IPO and LIPOC groups.
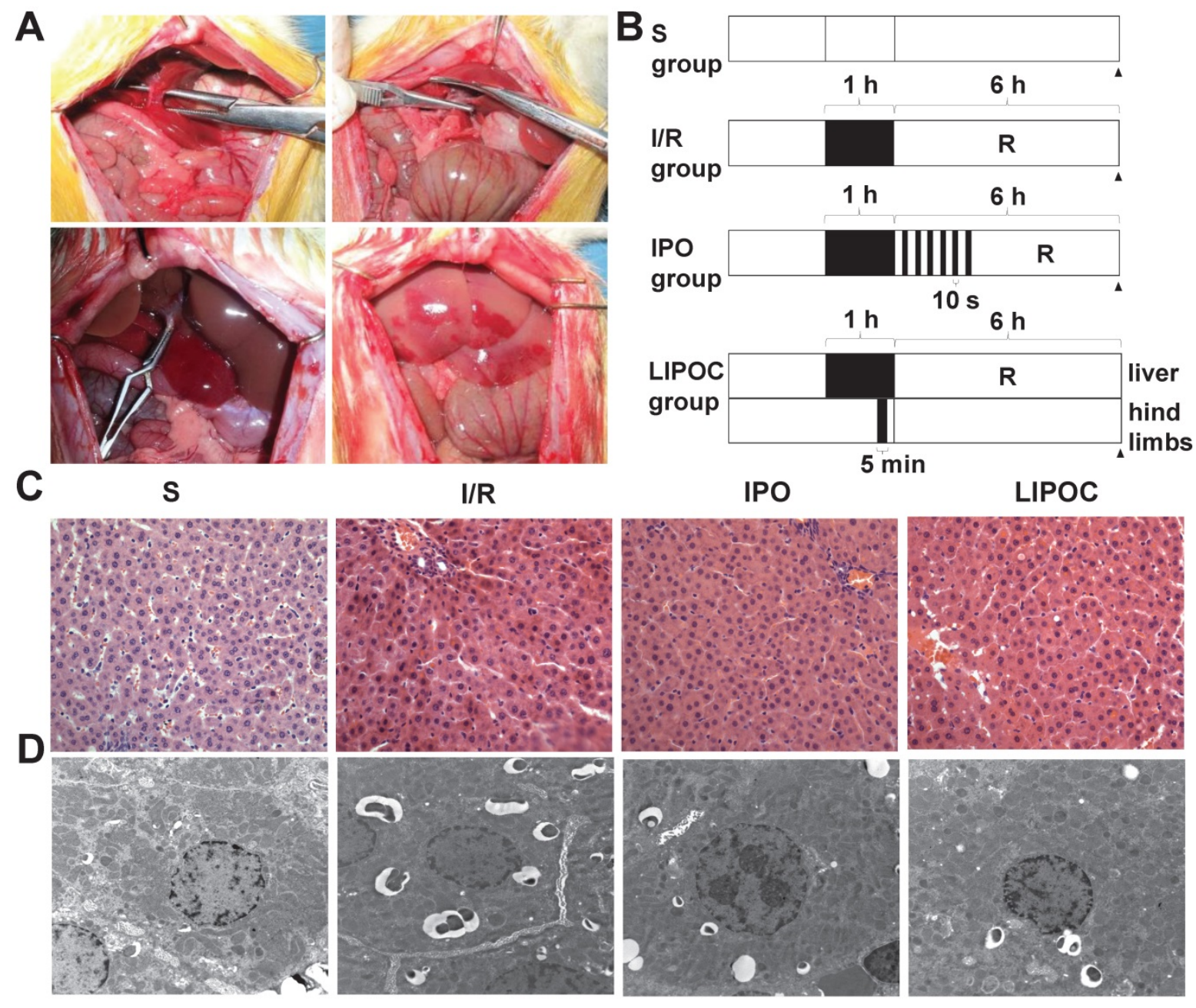

Figure 1. LIPOC ameliorates hepatic ischemia-reperfusion injury. (A) The establishment of the rat model for hepatic ischemia-reperfusion. Top left: anatomy of the left lobe, and the common running section of middle hepatic artery, portal vein, and bile duct; top right: noninvasive small blood vessels clamp was used to block the common running section to achieve a rat $70 \%$ hepatic ischemia model; bottom left: $70 \%$ liver warm ischemia for $1 \mathrm{~h}$; bottom right: reperfusion after $1 \mathrm{~h}$ liver warm ischemia. (B) The scheme of treatment design for four tested groups: S, I/R, IPO and LIPOC. R represents reperfusion. (C) Pathological changes in liver sections under light microscope (x400 magnification). (D) Pathological changes in liver sections under electron microscope (x10,000 magnification). 
A

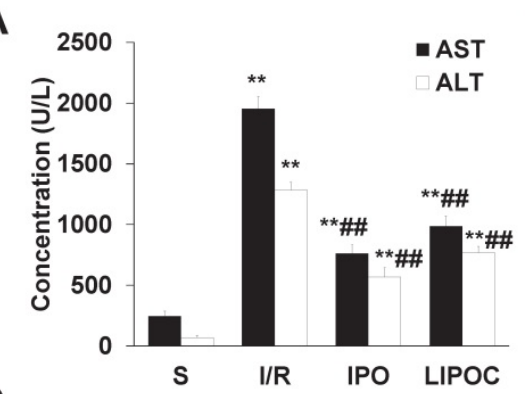

D

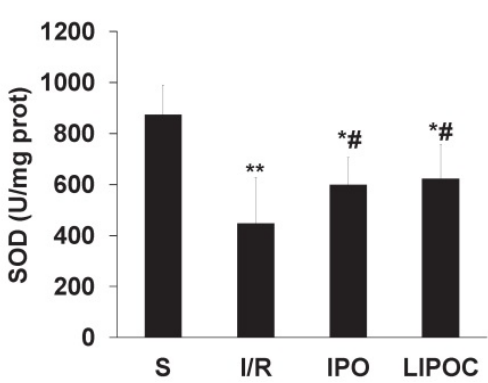

$B$

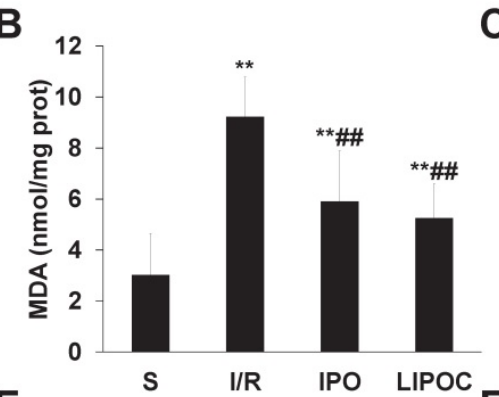

E

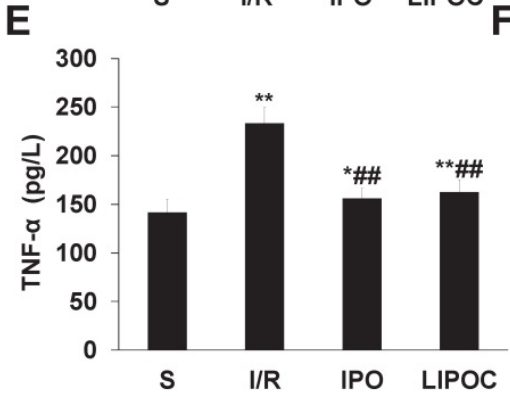

C
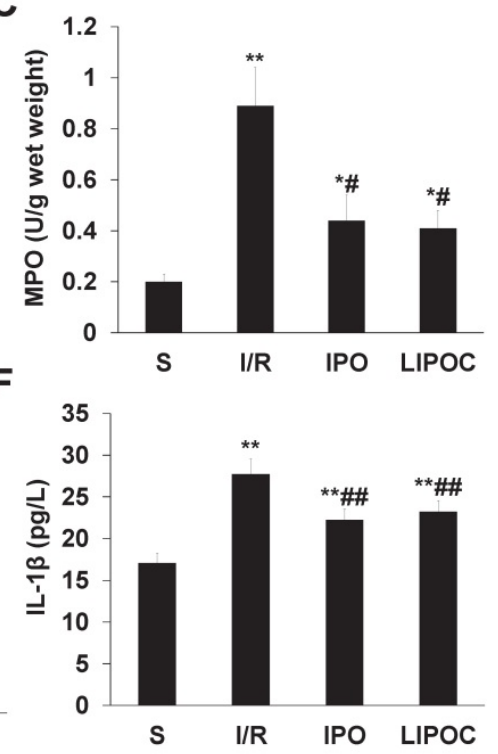

Figure 2. LIPOC reduces the synthesis of oxygen free radicals and the secretion of proinflammatory cytokines after reperfusion. (A) The serum levels of ALT and AST. (B) The levels of MDA in hepatic tissues (prot represents protein). (C) The levels of MPO in hepatic tissues. (D) The levels of SOD in hepatic tissues (prot represents protein). (E) The plasma levels of TNF- $\alpha$. (F) The plasma levels of IL-1 $\beta$. Compared to $S$ group: $* P<0.05$, $* * P<0.01$; compared to I/R group: $\# P<0.05, \ldots P<0.01$

\section{LIPOC reduces the synthesis of oxygen free radicals and the secretion of proinflammatory cytokines after reperfusion}

To analyze the mechanism for LIPOC's effects on hepatic ischemia-reperfusion injury, blood samples were collected from sub-hepatic vena cava of rats from different tested groups, and the serum levels of ALT and AST were measured by clinical automated analysis. Compared with $\mathrm{S}$ group, the plasma activities of ALT and AST (Fig. 2A) were increased significantly in I/R, IPO and LIPOC groups $(P<0.01)$; while compared with I/R group, the plasma activities of ALT and AST were decreased significantly in IPO and LIPOC groups $(P<0.01)$. No significant changes were found between IPO and LIPOC groups $(P>0.05)$. The concentrations of MDA, SOD and MPO in hepatic tissues were also measured, respectively. Compared with $S$ group, the MDA (Fig. 2B) and MPO (Fig. 2C) contents in hepatic tissues were increased, while the SOD contents (Fig. 2D) were decreased significantly in $\mathrm{I} / \mathrm{R}, \mathrm{IPO}$ and LIPOC groups $(P<0.05, P<0.01)$. Compared with $\mathrm{I} / \mathrm{R}$ group, the MDA and MPO contents were decreased, while the SOD contents were increased significantly in IPO and LIPOC groups $(P<0.05, P<0.01)$. In addition, compared with $S$ group, the plasma levels of tumor necrosis factor- $\alpha$ (TNF- $\alpha$ ) (Fig. 2E) and interleukin-1 $\beta$ (IL-1 $\beta$ ) (Fig. 2F) were increased significantly in I/R, IPO and LIPOC groups $(P<0.05, P<0.01)$; but compared with $\mathrm{I} / \mathrm{R}$ group, the plasma contents of TNF- $\alpha$ and IL-1 $\beta$ were decreased significantly in IPO and LIPOC groups $(P<0.01)$.

\section{LIPOC inhibits hepatocellular apoptosis and represses $\mathrm{Bax} / \mathrm{Bcl}-2$ ratio}

To investigate the protective effect of LIPOC on hepatocyte apoptosis, the apoptotic cells were detected by TUNEL staining using liver tissue of rats from each tested group. Compared with $S$ group, the hepatocellular apoptotic index (AI) was increased significantly in I/R, IPO and LIPOC groups $(P<0.01$, Fig. 3A-B). In contrast, when compared with $I / R$ group, the AIs were decreased significantly in IPO and LIPOC groups $(P<0.05$, Fig. 3A-B). To further understand the mechanism for LIPOC's effect on apoptosis, the expressions of $\mathrm{Bcl}-2$ and Bax protein in the liver tissues were detected by immuno-histochemistry. Compared with S group, the expression of Bcl-2 protein was increased significantly in $\mathrm{I} / \mathrm{R}, \mathrm{IPO}$ and LIPOC groups $(P<0.05$, Fig. $3 \mathrm{C})$. In comparison with I/R group, the expression of Bcl-2 protein was increased significantly in IPO and LIPOC groups $(P<0.05$, Fig. $3 C)$. In contrast, compared with $S$ group, the expression of Bax protein was increased significantly in I/R, IPO and LIPOC groups $(P<0.05$, Fig. 3D); while compared with $I / R$ group, the expression of Bax protein was decreased significantly in IPO and LIPOC groups $(P<0.05$, Fig. 3D).

\section{LIPOC enhances PI3K/Akt and MAPK signaling for hepatic protection}

To further investigate the mechanism of LIPOC on hepatic protection, we analyzed the effects of LIPOC on PI3K/Akt and ERKl/2 signaling. The rat 

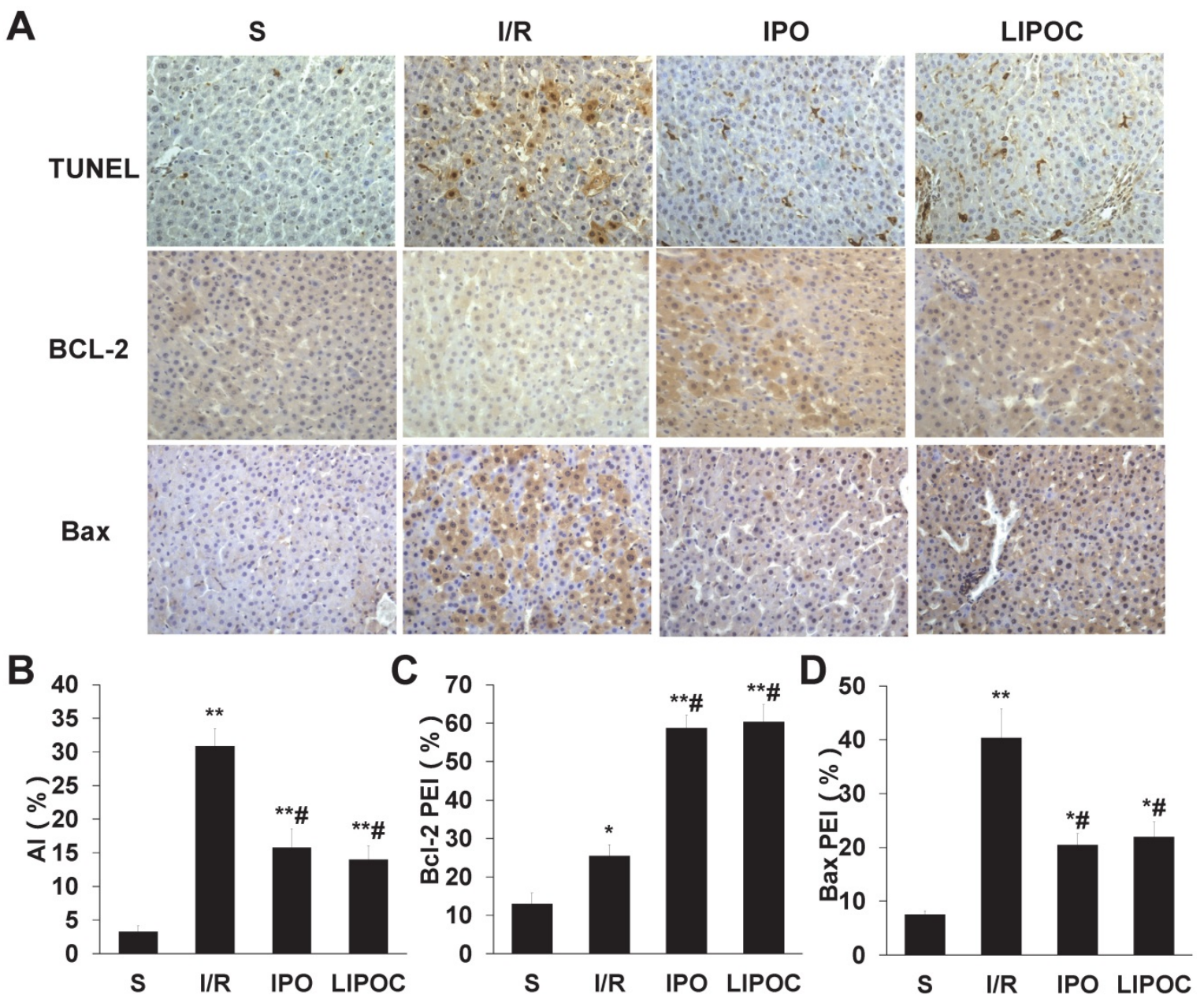

Figure 3. LIPOC inhibits hepatocellular apoptosis and represses Bax/Bcl-2 ratio. (A) Staining for TUNEL, Bcl-2, and Bax in liver tissue of rats from each tested group (x400 magnification). (B) The quantification of Al using TUNEL staining. (C) The quantification of Bcl-2 staining in each tested group. (D) The quantification of Bax staining in each tested group. Compared to $\mathrm{S}$ group: $* P<0.05$, $* * P<0.01$; compared to I/R group: $\# P<0.05$, \#P<0.01.

models of hepatic ischemia-reperfusion combined with the administration of PI3K inhibitor LY294002 and MAPK kinase inhibitor PD98059 were established. Forty-two healthy male Wistar rats were randomly divided into 7 groups: $S$ group, $L Y+S$ group, $\mathrm{PD}+\mathrm{S}$ group, I/R group, LIPOC group, LY+LIPOC group and PD+LIPOC group (Fig. 4A). The changes in the liver function were first assessed by analyzing the serum levels of ALT and AST. Compared with S group, the levels of ALT (Fig. 4B) and AST (Fig. 4C) were increased significantly in I/R, LIPOC, LY+LIPOC and PD+LIPOC groups $(P<0.01)$; while there was no difference among $S$ group, $L Y+S$ group and $\mathrm{PD}+\mathrm{S}$ group $(P>0.05)$. Compared with I/R group, the levels of ALT (Fig. 4B) and AST (Fig. 4C) were decreased significantly in LIPOC groups $(P<0.05, P<0.01)$; but there was no difference among $\mathrm{I} / \mathrm{R}, \mathrm{LY}+\mathrm{LIPOC}$ and PD+LIPOC groups $(P>0.05)$. Compared with LIPOC group, the levels of ALT (Fig. 4B) and AST (Fig. 4C) were increased significantly in LY+LIPOC and PD+LIPOC groups $(P<0.05, P<0.01)$.
No significant changes were found between LY+ LIPOC and PD+LIPOC groups $(P>0.05)$.

\section{The effects of LIPOC and RISK pathways on hepatocyte apoptosis and ultrastructural changes}

We then analyzed the role of RISK pathways in LIPOC's effects on hepatocyte apoptosis. Compared with $S$ group, the AI values of I/R group, LIPOC group, LY+LIPOC group and PD+LIPOC group were significantly higher than those of $S$ group $(P<0.01$, Fig. $5 \mathrm{~A} \& \mathrm{C})$. There was no significant difference between $S$ group and $\mathrm{PD}+S$ group and $\mathrm{LY}+\mathrm{S}$ group $(P>0.05$, Fig. $5 A \& C)$. The extent of increasing for $A I$ values in LIPOC group was significantly lower than I/R group $(P<0.05$, Fig. 5A\&C), while there was no significant difference between the LY+LIPOC group and the PD+LIPOC group with the I/R group $(P>0.05$, Fig. $5 \mathrm{~A} \& \mathrm{C})$. Compared with LIPOC group, AIs were significantly higher in the LY+LIPOC group and PD+LIPOC group $(P<0.05$, Fig. 5A\&C). There was no 
A

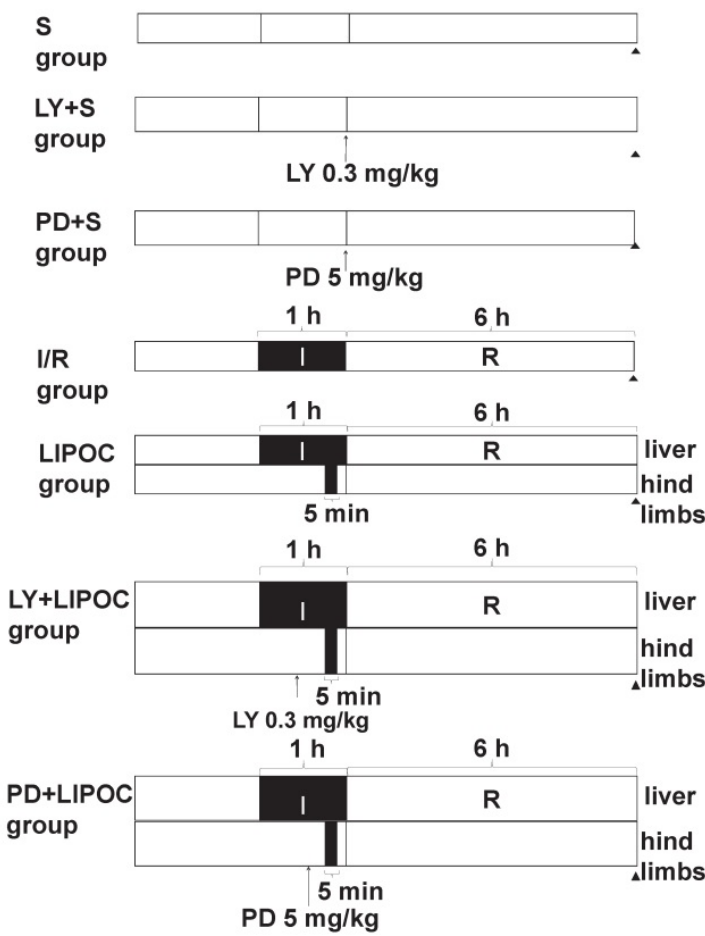

B

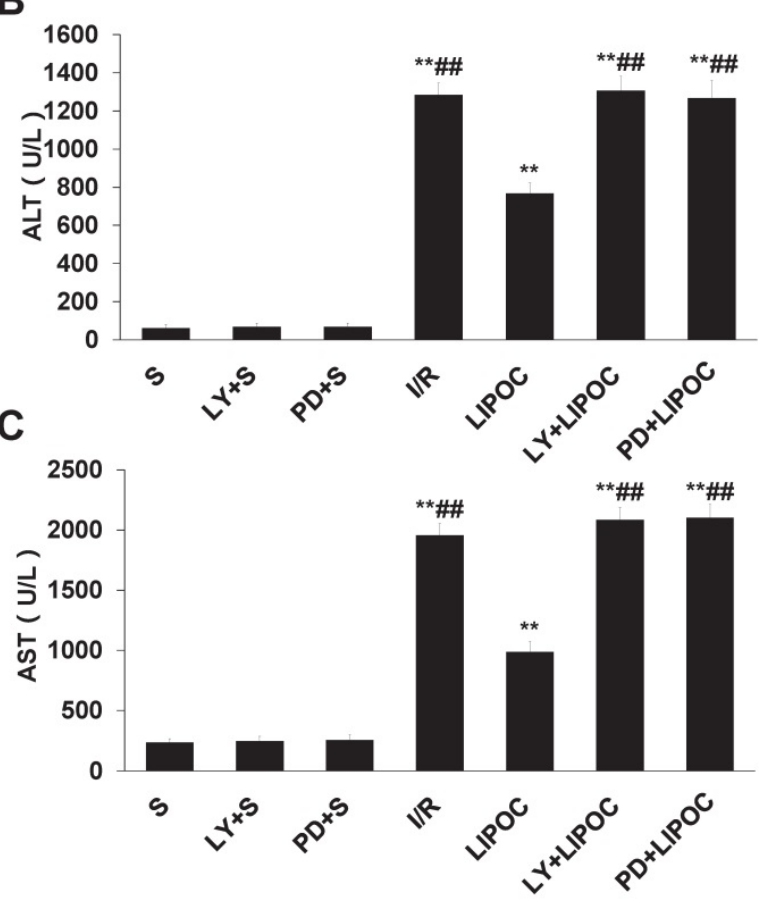

Figure 4. LIPOC enhances PI3K/Akt and MAPK signaling for hepatic protection. (A) The rat models of hepatic ischemia-reperfusion combined with the administration of PI3K inhibitor LY294002 and MAPK kinase inhibitor PD98059 were established. (B) The serum levels of ALT. (C) The serum levels of AST. Compared to S group: $* P<0.05, * * P<0.01$; compared to LIPOC group: $\# P<0.05,{ }^{\#} P<0.01$.

significant difference between LY+LIPOC group and PD+LIPOC group $(P>0.05$, Fig. 5A\&C). Under the electron microscopy, the structure of hepatocytes in $S$ group was normal, while the cells in $L Y+S$ group and $\mathrm{PD}+\mathrm{S}$ group didn't show obvious alterations compared to the $S$ group (Fig.5B). In LY+LIPOC and PD+LIPOC group, the structures of hepatocytes were very similar with that of $I / R$ group. There were many round inclusion bodies with high electron density in the cytoplasm. The inclusion bodies also contained necrotic mitochondria, and the tight junction structure was not clear (Fig. 5B).

\section{LY294002 and PD98059 antagonizes LIPOC increased $p$-Akt and p-ERK1/2}

To further understand the role of RISK signaling pathways in LIPOC's protective effects on hepatocytes, we analyzed the effects of LIPOC and its combination with LY294002 and PD98059 on the regulation of key molecules in RISK signaling. By using RT-qPCR analysis, we observed the expression of Akt mRNA and ERK1/2 mRNA in the liver tissues in each group, but there was no significant difference among each group (Supplemental Table 2). By using western blotting analysis, we observed a similar expression of Akt $(P>0.05)$ among different treatment groups, however, the expression of p-Akt was differentially regulated. Compared with $S$ group, the expression of p-Akt was increased significantly in I/ $R$ and LIPOC groups $(P<0.05, P<0.01$, Fig. 5D-E); while there was no difference among $S, L Y+S$ and LY+LIPOC groups $(P>0.05$, Fig. 5D-E). Similar effects were observed for Erk1/2 and p-Erk1/2 (Fig. 5D-F).

\section{Protective effect of LIPOC on hepatic ischemia-reperfusion injury in liver cancer patients undergoing hepatectomy}

To investigate the protective effect of LIPOC on HIRI in liver cancer patients undergoing hepatectomy, 16 liver cancer patients undergoing hepatectomy were randomly divided into control group and LIPOC group. LIPOC consisted of two 4-min tourniquet binding/4-min reperfusion cycles by automated cuff-inflator placed on the right leg after occlusion of hepatic hilum (Fig. 6A). In control group, the right leg blood-flow was not blocked (Fig. 6A), and there was no obvious difference about gender, age, weight, ASA grade, tumor size, occlusion time, operative time, fluid infusion volume, blood loss volume and blood transfusion volume during operation between control group and LIPOC group (Supplemental Table 1). The patients' venous blood samples were collected for the detection of liver function (including ALT, AST, TBIL, and ALB) before, at the end of and 1 day after the operation.

Compared with that of before operation, the levels of ALT (Fig. 6B), AST (Fig. 6C) and TBIL (Fig. 6D) were significantly higher in both groups after operation $(P<0.05, P<0.01)$. Compared with control group, the levels of ALT, AST and TBIL at the end of 
surgery and at 1 day after operation were significantly lower in LIPOC group $(P<0.05$, Fig. 6B-D). The levels of ALB (Fig. 6E) before operation were normal in both groups; compared with that before operation, the levels of ALB were significantly lower in both groups after operation $(P<0.05)$; while no significant change was found between control group and LIPOC group $(P>0.05)$. Therefore, LIPOC has protective effect on the ischemia-reperfusion injury of liver in patients with liver cancer during hepatectomy.

\section{Discussion}

In order to limit HIRI, blocking the hepatic portal circulation during liver surgery is a required and difficult step. The previously reported novel LIPOC approach has a protective effect on the IRI of heart and brain [13-16]. However, whether LIPOC clinically has a protective effect on IRI of the liver is largely unknown and has an urgent research significance and potential clinical application prospects. In this study, a $70 \% \mathrm{I} / \mathrm{R}$ model of rat liver was established to study the protective effect and molecular mechanism of LIPOC on HIRI. Through 16 cases of hepatocellular carcinoma resection, whether LIPOC has the protective effect on HIRI in hepatectomy was then investigated. The results show that LIPOC has a protective effect on hepatic ischemia-reperfusion injury in rats by reducing the elimination of superoxide dismutase, inhibiting neutrophil aggregation, and decreasing tumor necrosis factor- $\alpha$, interleukin-1 $\beta$ and other inflammatory cytokines. Moreover, LIPOC can inhibit the apoptosis of hepatocytes induced by $\mathrm{I} / \mathrm{R}$ injury possibly by promoting the expression of Bcl-2 and inhibiting the expression of pro-apoptotic protein Bax. Further, LIPOC resulted in a higher level of phosphorylation of Akt and ERK1/2. The use of PI3K inhibitor LY294002 and ERK1/2 blocker PD98059 did not only inhibit the phosphorylation of Akt caused by LIPOC but also made the injury protection of liver I/R disappear. Through the observation of patients with liver cancer, we found that short-term unilateral lower limb ischemic treatment significantly repressed the increased amplitude of ALT, AST, and TBIL in the early postoperative of liver resection, and reduced reperfusion injury to the ischemic liver in liver resection.

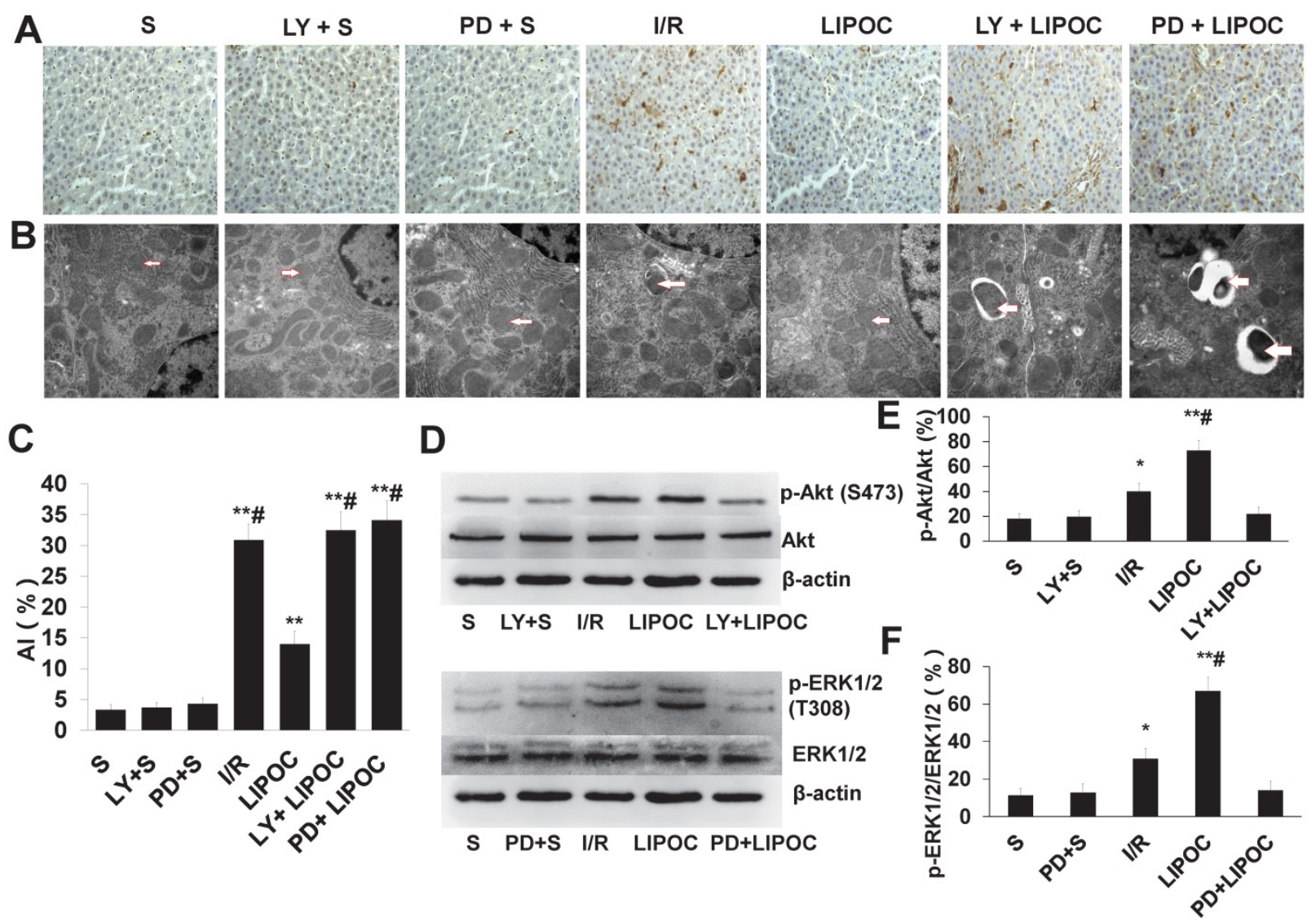

Figure 5. The effects of LIPOC and RISK pathways on hepatocyte apoptosis and ultrastructural changes. (A) TUNEL staining for liver tissue of rats from each tested group ( $\times 400$ magnification). (B) Pathological changes in liver sections under electron microscope ( $\times 30,000$ magnification). The structural changes of mitochondria are indicated by the arrows. (C) The quantification of Al using TUNEL staining. Compared to $S$ group: $* * P<0.01 ;$ compared to LIPOC group: \#P<0.05. (D) Western blotting analysis for the expression and phosphorylation of Akt and ERK1/2 among different treatments. (E) Quantification of Akt expression and phosphorylation with different treatments. (F) Quantification of ERK1/2 expression and phosphorylation with different treatments. 

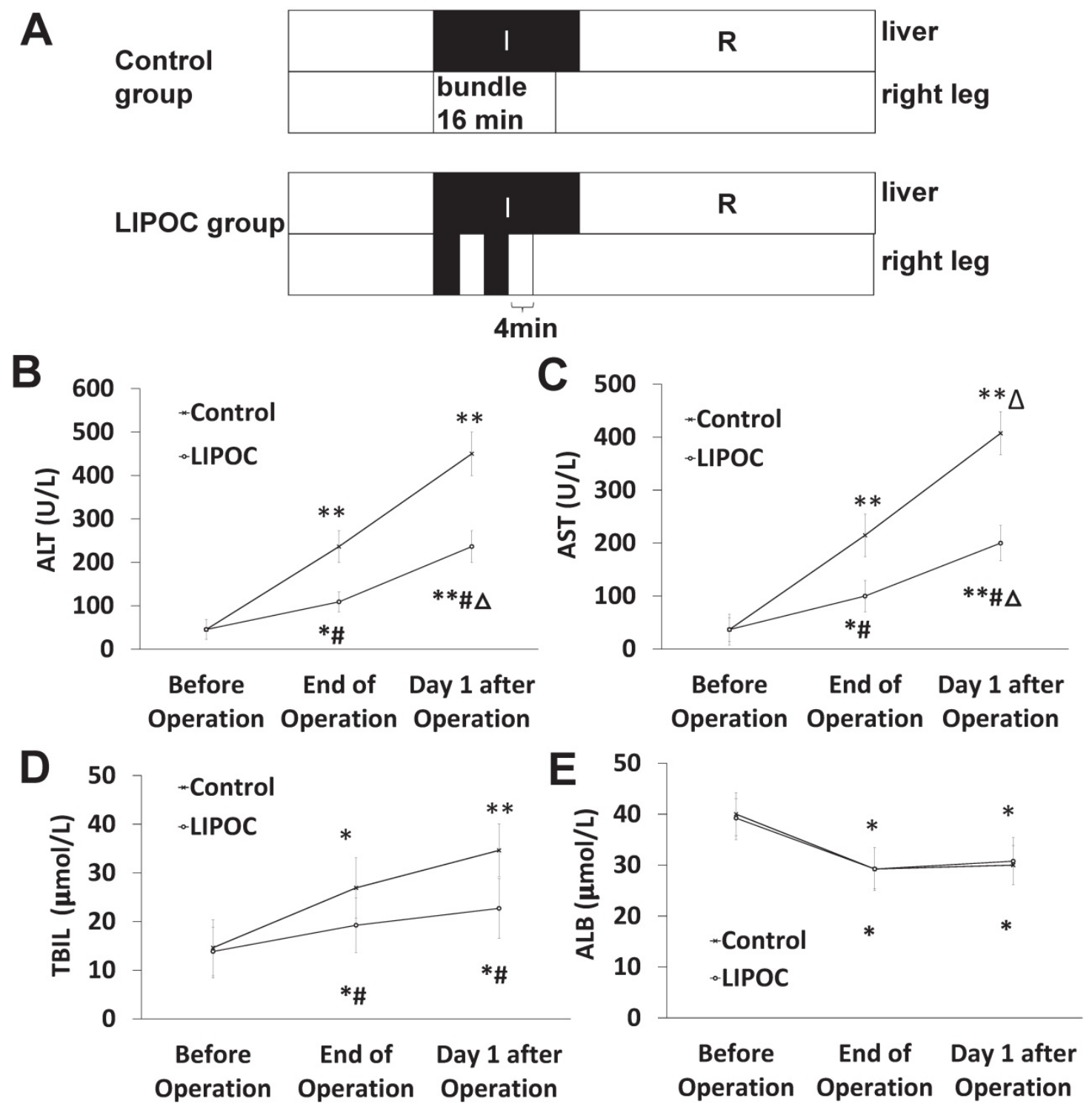

Figure 6. Protective effect of LIPOC on hepatic ischemia-reperfusion injury in liver cancer patients undergoing hepatectomy. (A) The scheme of treatment design for 16 liver cancer patients undergoing hepatectomy. Patients were randomly divided into control group and LIPOC group. (B) The levels of ALT before operation, at the end of operation, and after operation. (C) The levels of AST before operation, at the end of operation, and after operation. (D) The levels of TBIL before operation, at the end of operation, and after operation (E) The levels of ALB before operation, at the end of operation, and 1 day after operation. Compared to levels before operation: $* P<0.05,{ }^{*} * P<0.01$; compared to control group: $\# P<0.05$, \#P<0.01; compared to levels at the end of operation: ${ }^{\circ} P<0.05$.

The LIPOC method in hepatectomy can reduce the reperfusion injury in the ischemic liver. In recent years, several experimental I/R injury models have been improvised to test the efficacy of post-conditioning maneuvers on attenuating liver I/ $\mathrm{R}$ injury [32]. The vast majority of these studies were performed by intermittent sequential interruptions of blood flow in the early phase of reperfusion of the operative organ or another remote vital organ, e.g. kidney $[32,33]$. In contrast, as a non-invasive method, LIPOC shows obvious superiority by simply applying transient I/R on limb, not influencing operation procedures, and without extending the operation time. Although LIPOC has been demonstrated effective on protecting heart and brain from ischemic injury [34-36], there is limited information about its effects on liver protection. Prior to our study, there was no previous clinical data to demonstrate the efficacy of LIPOC or other remote lower limb ischemia-reperfusion on liver protection using both rat model and human pilot trial.

Since there is no report for the clinical application of LIPOC in liver surgery yet, the treatment of this study was mainly referred to the method used by LIPOC in cardiac surgery. For example, Andreka et al. [34] intermittently gave limb 4 cycles of 5-min ischemia/ 5-min reperfusion during myocardial ischemia resulted in myocardial protection. Loukogeorgakis et al. [37] used a sphygmomanometer induced transient ischemia to 
trigger LIPOC in the lower limbs, and compared the contributions of various factors, such as the number of cycles or the duration of ischemia, on the protective effect of LIPOC. The results showed that 2 times of 5-min ischemia / 5-min reperfusion during ischemia could reduce endothelial cell injury. However, the use of LIPOC for HIRI may not exactly follow the method for operations on heart, because of the hindrance time limit for liver. A large number of clinical trials have shown that the safe hilar occlusion time is between 15 $\sim 20 \mathrm{~min}$ [38-40]. In this study, according to the Loukogeorgakis et al.'s method, under the premise of ensuring the number of treatment, we reduced $1 \mathrm{~min}$ of each treatment to ensure that the second ischemic treatment of lower limb has been completed before the opening of the hepatic vascular. Therefore, we performed unilateral lower limb after the hilar block for 2 times of 4-min ischemia/ 4-min reperfusion (a total of 16 min treatment). The results of this study show that the LIPOC method we used in hepatectomy can reduce the reperfusion injury in the ischemic liver. In this study, we only performed unilateral lower limb LIPOC, whether bilateral lower extremity LIPOC has a better protective effect or not requires further study. In addition, the inflation pressure of 200 $\mathrm{mmHg}$ was used in the study. With the premise of ensuring the safety of the limbs, whether increasing the inflation pressure can result in a better liver protective effect also awaits to be explored in the future.

LIPOC enhances PI3K/Akt and MAPK signaling for hepatic protection. It has been reported that PI3K/Akt signaling pathway is involved in the protection of HIRI. Carini et al. [41] found that liver cells after hypoxia preconditioning active PI3K and regulate PKC-dependent signaling pathway, indicating that PI3K plays a vital role in hypoxia tolerance of liver cells. Ke et al. [42] found that PI3K/Akt inhibited IL-12 production and enhanced the function of anti-apoptotic gene Bcl-2/Bcl-XL to reduce the damage to the liver cell. In our study, compared with LIPOC group, the levels of serum ALT and AST were significantly increased in LY+LIPOC group, and the improvement of liver function by LIPOC was significantly inhibited by LY294002. Electron microscopic pathology also showed that LY294002 could impair the effects of LIPOC in relieving liver cell structure damage. LY+LIPOC group has a significantly higher apoptotic index than LIPOC group, suggesting that LY294002 could contradict the inhibitory effect of LIPOC on hepatocyte apoptosis. These results suggest that the activation of PI3K/Akt signaling pathway mediates the protective effect of LIPOC on HIRI and specific PI3K blocker LY294002 significantly inhibits the protective effect of LIPOC on the liver. We also analyzed the protein expression of key molecules in PI3K signaling pathway. After $6 \mathrm{~h}$ of reperfusion, the expression of Akt was not changed, while the phosphorylation of Akt was increased by LIPOC. The use of PI3K inhibitor LY294002 before LIPOC treatment did not only inhibit the phosphorylation of Akt induced by LIPOC, but also the protective effect of LIPOC on HIRI, indicating that PI3K activation plays critical roles in liver's self-protection of IRI.

Studies have shown that in the IRI of vital organs, activation of ERK protein kinase can be observed at the early stage of injury [20,43]. However, the expression and phosphorylation patterns may be distinct in different tissues and organs. ERKs are thought to have anti-apoptotic effects in the heart and other organs' I/Rs and have a protective effect on I/R organs [44], though there are limited studies on ERK in the liver I/R, and the role of ERK1/2 in LIPOC has not yet been determined. This study investigated the role of ERK1/2 signaling pathway in LIPOC protection against HIRI. Compared with LIPOC group, serum ALT and AST levels were significantly increased in PD+LIPOC group, and PD98059 significantly inhibited the improvement of liver function in LIPOC group. Electron microscopic pathology also showed that PD98059 could diminish the effects of LIPOC in relieving liver structure damage. The apoptotic index of PD+LIPOC group was significantly higher than that of LIPOC group, suggesting that PD98059 could lessen the inhibitory effect of LIPOC on hepatocyte apoptosis. These results suggest that the protective effect of LIPOC was significantly inhibited by the specific ERK1/2 blocker PD98059 on the liver, indicating that the protective effect of LIPOC on HIRI was mediated by the activation of ERK1/2 signaling pathway. In this study, we found that liver protection of LIPOC would be abolished even if only one of the two RISK pathways (PI3K or ERK1/2) was blocked, suggesting that the two signaling pathways are both required for the protective effect of LIPOC on liver. It is not clear, however, how PI3K and ERK1/2 exert the protective effects after activated by LIPOC. Studies have reported that $\mathrm{MPTP}$ may be a terminal effector for multiple signal transduction pathways for organ protection [45]. Whether PI3K and ERK1/2 also act via $\mathrm{mPTP}$ in the liver or not requires further investigation.

\section{Conclusions}

In summary, by analyzing the data from the liver $\mathrm{I} / \mathrm{R}$ rat model and primary liver cancer patients with hepatectomy, we show that LIPOC in hepatectomy surgery may be able to reduce the ischemic liver 
reperfusion injury. We also demonstrated that the RISK signaling pathways composed of PI3K/Akt and ERK1/2 signaling are involved in the protective effects of LIPOC on hepatic ischemia-reperfusion injury in rats. Our study provides evidence supporting the clinical application of LIPOC in HIRI in order to reduce the perioperative complications for hepatectomy and improve surgical outcomes for patients with primary liver cancer and/or other liver diseases.

\section{Abbreviations}

AI: apoptosis index; ALT: alanine aminotransferase; AST: aspartic transaminase; HIRI: hepatic ischemia-reperfusion injury; IPC: ischemic preconditioning; I/R: ischemia-reperfusion; IRI: ischemia-reperfusion injury; LIPOC: limb ischemic post-conditioning; MPO: myeloperoxidase; PEI: protein expression index; PI3K/Akt: phosphatidylinositol-3-kinase/protein kinase B; PMN: polymorphonuclear leukocytes; RIPOC: remote ischemic post-conditioning; RISKs: reperfusion injury salvage kinases; SD: Sprague-Dawley; SOD: superoxide dismutase; TBA: thiobarbituric acid.

\section{Supplementary Material}

Supplementary tables.

http://www.ijbs.com/v14p2037s1.pdf

\section{Acknowledgements}

This study was supported by the Science and Technology Project of Social Development of Shaanxi Province, China (No. 2012K13-01-05), the Science and Technology Project of Social Development of Shaanxi Province, China (No. 2016SF-139), the Clinical Research Award of the First Affiliated Hospital of $X^{\prime}$ 'an Jiaotong University, China (No. XJTU1AHCR2014-035), and Baylor Scott \& White Health Funds.

\section{Author Contributions}

YG, SZ, EW, YL, XH conceived and designed the experiments. $Y G$ and $S Z$ performed the experiments. YG, SZ, FW, YZ, SS, YL, EW analyzed the data. YG, $\mathrm{YL}$ and $\mathrm{XH}$ contributed reagents/materials/analysis tools. YG, SZ, FW, YZ, SS, JHH, EW, DQ, YL, and XH contributed to manuscript preparation, writing, and revision.

\section{Ethics Approval}

The human subject studies were approved by the ethical standards committee of X'an Jiaotong University. Written informed consents were obtained from the patients participating in the study.

\section{Competing Interests}

The authors have declared that no competing interest exists.

\section{References}

1. Siegel RL, Miller KD, Jemal A. Cancer Statistics, 2017. CA Cancer J Clin. 2017; 67: 7-30.

2. Zhai Y, Petrowsky H, Hong JC, Busuttil RW, Kupiec-Weglinski JW. Ischaemia-reperfusion injury in liver transplantation--from bench to bedside. Nat Rev Gastroenterol Hepatol. 2013; 10: 79-89.

3. Murry CE, Jennings RB, Reimer KA. Preconditioning with ischemia: a delay of lethal cell injury in ischemic myocardium. Circulation. 1986; 74: 1124-36.

4. Clavien PA, Yadav S, Sindram D, Bentley RC. Protective effects of ischemic preconditioning for liver resection performed under inflow occlusion in humans. Ann Surg. 2000; 232: 155-62.

5. Chu MJ, Vather R, Hickey AJ, Phillips AR, Bartlett AS. Impact of ischemic preconditioning on outcome in clinical liver surgery: a systematic review. Biomed Res Int. 2015; 2015: 370451.

6. Boyko VV, Pisetska ME, Tyshchenko OM, Skoryi DI, Kozlova TV, Gorgol NI, et al. Role of ischemic preconditioning in hepatic ischemia-reperfusion injury. Hepatobiliary Surg Nutr. 2014; 3: 179-84.

7. Oxman T, Arad M, Klein R, Avazov N, Rabinowitz B. Limb ischemia preconditions the heart against reperfusion tachyarrhythmia. Am J Physiol. 1997; 273: H1707-12.

8. Veighey $\mathrm{K}$, Macallister RJ. Clinical applications of remote ischemic preconditioning. Cardiol Res Pract. 2012; 2012: 620681.

9. Meng R, Asmaro K, Meng L, Liu Y, Ma C, Xi C, et al. Upper limb ischemic preconditioning prevents recurrent stroke in intracranial arterial stenosis. Neurology. 2012; 79: 1853-61.

10. Kharbanda RK, Mortensen UM, White PA, Kristiansen SB, Schmidt MR, Hoschtitzky JA, et al. Transient limb ischemia induces remote ischemic preconditioning in vivo. Circulation. 2002; 106: 2881-3.

11. Zhao ZQ, Corvera JS, Halkos ME, Kerendi F, Wang NP, Guyton RA, et al. Inhibition of myocardial injury by ischemic postconditioning during reperfusion: comparison with ischemic preconditioning. Am J Physiol Heart Circ Physiol. 2003; 285: H579-88.

12. Kerendi F, Kin H, Halkos ME, Jiang R, Zatta AJ, Zhao ZQ, et al. Remote postconditioning. Brief renal ischemia and reperfusion applied before coronary artery reperfusion reduces myocardial infarct size via endogenous activation of adenosine receptors. Basic Res Cardiol. 2005; 100: 404-12.

13. Lavi S, D'Alfonso S, Diamantouros P, Camuglia A, Garg P, Teefy P, et al. Remote ischemic postconditioning during percutaneous coronary interventions: remote ischemic postconditioning-percutaneous coronary intervention randomized trial. Circ Cardiovasc Interv. 2014; 7: 225-32.

14. Song X, Zhang N, Xu H, Cao L, Zhang H. Combined preconditioning and postconditioning provides synergistic protection against liver ischemia-reperfusion injury. Int J Biol Sci. 2012; 8: 707-18.

15. Zong Y, Jiang L, Zhang M, Zhou F, Qi W, Li S, et al. Limb remote ischemic postconditioning protects cerebral ischemia from injury associated with expression of HIF-1alpha in rats. BMC Neurosci. 2015; 16: 97.

16. Crimi G, Pica S, Raineri C, Bramucci E, De Ferrari GM, Klersy C, et al. Remote ischemic post-conditioning of the lower limb during primary percutaneous coronary intervention safely reduces enzymatic infarct size in anterior myocardial infarction: a randomized controlled trial. JACC Cardiovasc Interv. 2013; 6: 1055-63.

17. Hausenloy DJ, Yellon DM. Reperfusion injury salvage kinase signalling: taking a RISK for cardioprotection. Heart Fail Rev. 2007; 12: 217-34.

18. Hausenloy DJ, Tsang A, Yellon DM. The reperfusion injury salvage kinase pathway: a common target for both ischemic preconditioning and postconditioning. Trends Cardiovasc Med. 2005; 15: 69-75.

19. Hausenloy DJ, Yellon DM. New directions for protecting the heart against ischaemia-reperfusion injury: targeting the Reperfusion Injury Salvage Kinase (RISK)-pathway. Cardiovasc Res. 2004; 61: 448-60.

20. Hausenloy DJ, Barrabes JA, Botker HE, Davidson SM, Di Lisa F, Downey J, et al. Ischaemic conditioning and targeting reperfusion injury: a 30 year voyage of discovery. Basic Res Cardiol. 2016; 111: 70.

21. Hausenloy DJ, Yellon DM. Ischaemic conditioning and reperfusion injury. Nat Rev Cardiol. 2016; 13: 193-209.

22. Shamloo M, Rytter A, Wieloch T. Activation of the extracellular signal-regulated protein kinase cascade in the hippocampal CA1 region in a rat model of global cerebral ischemic preconditioning. Neuroscience. 1999; 93: 81-8.

23. Shamloo M, Wieloch T. Changes in protein tyrosine phosphorylation in the rat brain after cerebral ischemia in a model of ischemic tolerance. J Cereb Blood Flow Metab. 1999; 19: 173-83.

24. Pignataro G, Scorziello A, Di Renzo G, Annunziato L. Post-ischemic brain damage: effect of ischemic preconditioning and postconditioning and identification of potential candidates for stroke therapy. FEBS J. 2009; 276: 46-57. 
25. Yan Y, Li G, Tian X, Ye Y, Gao Z, Yao J, et al. Ischemic preconditioning increases GSK-3beta/beta-catenin levels and ameliorates liver ischemia/reperfusion injury in rats. Int J Mol Med. 2015; 35: 1625-32.

26. Pan MX, Zhang Y, Li AH, Gao Y. Protective effect of protein kinase $\mathrm{C}$ and mitogen-activated protein kinases and its mechanism in liver ischemic preconditioning. Nan Fang Yi Ke Da Xue Xue Bao. 2006; 26: 1188-1193.

27. Guo JY, Yang T, Sun XG, Zhou NY, Li FS, Long D, et al. Ischemic postconditioning attenuates liver warm ischemia-reperfusion injury through Akt-eNOS-NO-HIF pathway. J Biomed Sci. 2011; 18: 79.

28. Dal Ponte C, Alchera E, Follenzi A, Imarisio C, Prat M, Albano E, et al. Pharmacological postconditioning protects against hepatic ischemia/reperfusion injury. Liver Transpl. 2011; 17: 474-82.

29. Tsung A, Sahai R, Tanaka H, Nakao A, Fink MP, Lotze MT, et al. The nuclear factor HMGB1 mediates hepatic injury after murine liver ischemia-reperfusion. J Exp Med. 2005; 201: 1135-43.

30. Mendes-Braz M, Elias-Miro M, Jimenez-Castro MB, Casillas-Ramirez A, Ramalho FS, Peralta C. The current state of knowledge of hepatic ischemia-reperfusion injury based on its study in experimental models. J Biomed Biotechnol. 2012; 2012: 298657.

31. Li CM, Zhang $\mathrm{XH}, \mathrm{Ma} \mathrm{XJ}$, Luo M. Limb ischemic postconditioning protects myocardium from ischemia-reperfusion injury. Scand Cardiovasc J. 2006; 40; 312-7.

32. Theodoraki K, Karmaniolou I, Tympa A, Tasoulis MK, Nastos C, Vassiliou I, et al. Beyond Preconditioning: Postconditioning as an Alternative Technique in the Prevention of Liver Ischemia-Reperfusion Injury. Oxid Med Cell Longev. 2016; 2016: 8235921.

33. Ricca L, Lemoine A, Cauchy F, Hamelin J, Sebagh M, Esposti DD, et al. Ischemic Postconditioning of the Liver Graft in Adult Liver Transplantation. Transplantation. 2015; 99: 1633-43

34. Andreka G, Vertesaljai M, Szantho G, Font G, Piroth Z, Fontos G, et al. Remote ischaemic postconditioning protects the heart during acute myocardial infarction in pigs. Heart. 2007; 93: 749-52.

35. Chen G, Thakkar M, Robinson C, Dore S. Limb Remote Ischemic Conditioning: Mechanisms, Anesthetics, and the Potential for Expanding Therapeutic Options. Front Neurol. 2018; 9: 40.

36. Cheng X, Zhao H, Yan F, Tao Z, Wang R, Han Z, et al. Limb remote ischemic post-conditioning mitigates brain recovery in a mouse model of ischemic stroke by regulating reactive astrocytic plasticity. Brain Res. 2018; 1686: 94-100.

37. Loukogeorgakis SP, Williams R, Panagiotidou AT, Kolvekar SK, Donald A, Cole TJ, et al. Transient limb ischemia induces remote preconditioning and remote postconditioning in humans by a K(ATP)-channel dependent mechanism. Circulation. 2007; 116: 1386-95.

38. Belghiti J, Noun R, Malafosse R, Jagot P, Sauvanet A, Pierangeli F, et al. Continuous versus intermittent portal triad clamping for liver resection: a controlled study. Ann Surg. 1999; 229: 369-75.

39. Chouillard EK, Gumbs AA, Cherqui D. Vascular clamping in liver surgery: physiology, indications and techniques. Ann Surg Innov Res. 2010; 4: 2.

40. Lei DX, Peng $\mathrm{CH}$, Peng SY, Jiang XC, Wu YL, Shen HW. Safe upper limit of intermittent hepatic inflow occlusion for liver resection in cirrhotic rats. World J Gastroenterol. 2001; 7: 713-7.

41. Carini R, Grazia De Cesaris M, Splendore R, Baldanzi G, Nitti MP, Alchera E, et al. Role of phosphatidylinositol 3-kinase in the development of hepatocyte preconditioning. Gastroenterology. 2004; 127: 914-23.

42. Ke B, Shen XD, Kamo N, Ji H, Yue S, Gao F, et al. beta-catenin regulates innate and adaptive immunity in mouse liver ischemia-reperfusion injury. Hepatology. 2013; 57: 1203-14

43. Friguls B, Petegnief V, Justicia C, Pallas M, Planas AM. Activation of ERK and Akt signaling in focal cerebral ischemia: modulation by TGF-alpha and involvement of NMDA receptor. Neurobiol Dis. 2002; 11: 443-56.

44. Abe J, Baines CP, Berk BC. Role of mitogen-activated protein kinases in ischemia and reperfusion injury : the good and the bad. Circ Res. 2000; 86: 607-9.

45. Perrelli MG, Pagliaro P, Penna C. Ischemia/reperfusion injury and cardioprotective mechanisms: Role of mitochondria and reactive oxygen species. World J Cardiol. 2011; 3: 186-200. 